\title{
Diacronie
}

Studi di Storia Contemporanea

$\mathrm{N}^{\circ} 18,2 \mid 2014$

Le esposizioni: propaganda e costruzione identitaria

\section{Les pavillons russes aux Expositions Universelles du XIXe siècle: expression de l'identité qui n'a jamais existé}

\section{Olga Kazakova}

\section{OpenEdition}

\section{Journals}

Édition électronique

URL : http://journals.openedition.org/diacronie/1411

DOI : 10.4000/diacronie. 1411

ISSN : 2038-0925

Éditeur

Association culturelle Diacronie

Référence électronique

Olga Kazakova, "Les pavillons russes aux Expositions Universelles du XIXe siècle: expression de l'identité qui n'a jamais existé », Diacronie [En ligne], № 18, 2 | 2014, document 6, mis en ligne le 01 juin 2014, consulté le 01 mai 2019. URL : http://journals.openedition.org/diacronie/1411 ; DOI : 10.4000/ diacronie. 1411 


\title{
Diacronie
}

6/

\section{Les pavillons russes aux Expositions}

\section{Universelles du XIXe siècle: expression de} l'identité qui n'a jamais existé

\author{
Olga KAZAKOVA*
}

La Russie a pris part presque à toutes les Expositions Universelles de la deuxième moitié du XIXe siècle. Elle a commencé par les sections du bâtiment principal, puis elle a élargi sa présence aux expositions avec des édifices thématiques et de grands pavillons nationaux. La réaction des visiteurs de ces Expositions influençait l'établissement de l'esthétique particulière des pavillons russes suivant le style folklorique. Ils créent l'idée de la Russie fausse au fond mais intéressante et familière aux Européens, celle du pays oriental et préindustriel.

\section{Introduction}

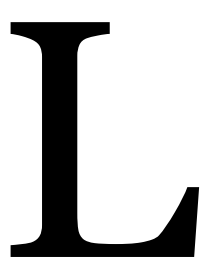

es Expositions Universelles ont été conçues comme une manifestation des progrès scientifiques et techniques de l'humanité et des échanges de connaissances. Leur conception avait un caractère universaliste. Pour l'exposition centrale on édifiait spécialement une construction en métal dont la carcasse était remplie de verre. Dès le «Crystal Palace» de Joseph Paxton (1851) on attribuait à tous les pavillons des expositions suivantes un air de «palais». Ces constructions cyclopéennes en fer et en verre de design futuriste sont devenues un attribut infaillible et un symbole des Expositions Universelles du XIX ${ }^{\mathrm{e}}$ siècle. 
Cependant une telle exposition en tant que scientifique et technique ne pouvait pas être universelle ni compter sur un afflux massif de visiteurs. La révolution industrielle n'a pas encore gagné la plupart des pays d'Asie, d'Afrique, d'Amérique latine. Les visiteurs-spécialistes y compris des ingénieurs et des hommes d'affaires n'auraient pas fait récupérer les dépenses des organisateurs. C'est pourquoi à partir de l'Exposition de Londres on a vu apparaître des sections nationales présentant des minéraux locaux, des échantillons de la production agricole et artisanale destinés à attirer le public ordinaire. C'est ainsi que la conception universaliste a commencé à subir une mutation vers l'exotisme éthno-géographique divertissant. Dans cette nouvelle approche les participants ont déclaré intentionnellement fort leur individualité, leur identité unique. Au début les pays ont réservé le rôle des dominantes visuelles des sections aux faussesfaçades («entrées-vestibules») sous la forme des copies réduites des célèbres cathédrales ou palais.

L'Exposition Universelle de Paris de 1878 s'avère un tournant décisif car elle a insisté sur une condition sine qua non - présentation du pavillon national particulier. La plupart des pays ont copié pour la construction de leurs pavillons les exemples exceptionnels de l'architecture nationale. Il était difficile aux participants de remplir les pavillons car les produits d'industrie, de science, de culture étaient exposés dans les sections thématiques. Par conséquence les pavillons nationaux se transformaient en musées régionaux - récits de la géographie (cartes, photographies, tableaux, maquettes), de l'ethnographie (mannequins en costumes populaires, éléments d'habitation, scènes de la vie quotidienne) et de l'histoire. Ainsi les façades et les intérieurs des pavillons nationaux se complétaient présentant une image pittoresque du pays.

\section{2. État de l'art}

Le sujet des pavillons aux Expositions Universelles a la place très modeste dans l'historiographie de la participation de la Russie aux ces manifestations. On peut la diviser en trois parties: le point de vue esthétique, historique et idéologique.

Dans l'histoire de l'art le sujet étudié a l'historiographie plus abondante, parce que certains auteurs des pavillons, notamment, Ivan Ropete, sont des architectes de premier ordre ou très à la mode à son époque et même aujourd'hui. L'opinion de Vladimir Stassov, célèbre critique des beaux-arts et promoteur passionné du style 
pseudo-russe («le style de coq») de la deuxième moitié du XIX siècle ${ }^{1}$, domine dans l'historiographie, en cédant une fois, au début du XX siècle, à l'autre critique des beauxarts et leader de courant de l'art-déco russe, Alexandre Benois (la plus complète bibliographie sur le site Héritage d'Alexandre Nikolaevich Benois ${ }^{2}$. Si le premier (et ses partisans contemporains) exigeait l'originalité nationale des pavillons à tout prix, le deuxième défendait les principes occidentaux de l'art moderne incarnés dans la conception des pavillons. Dans ces débats esthétiques la question de l’identité vraiment russe des pavillons était substituée par l'image artificielle de la Russie pour les étrangers. Pourtant l'historiographie de l'art décrit bien et apprécie la contribution de l'architecture des pavillons des Expositions dans le développement des beaux-arts en Russie. Une des dernières tentatives de ce genre est l'intervention de A. Avetisian Traditions de l'architecture russe dans l'architecture des pavillons de la Russie aux Expositions Universelles3.

Dans l'histoire générale de la Russie le sujet étudié a trouvé sa place dans l'histoire de l'industrie et du progrès technique. L'oeuvre classique de ce genre est «Parade des Expositions Universelles» (1990) de Nikolay Mezenine4, parmi des dernières publications on peut indiquer le livre Histoire des Expositions Universelles (2008) de Valerii Chpakov5. Cette historiographie a deux traits particuliers. D'un côté, elle ne sépare pas l'histoire des Expositions de l'époque de la monarchie russe du temps de l'URSS, considère la participation de la Russie comme le mouvement commun et triomphant du génie innovateur national. De l'autre côté, elle s'intéresse au contenu des pavillons, à la technologie de la construction des bâtiments (l'isba sans clous de l'Expo-1867, le palais en brique de l'Expo-1900), plus qu'à leurs extérieur, sans dire à leurs sens identitaire.

L’intérêt actuel vers le sujet étudié est stimulé par la publicité de Ekaterinburg pour le concours de l'Expo-2020. Les efforts des organisateurs et leurs PR-agences attirent l'attention des chercheurs et du public vers l'histoire de la participation russe (avant tout, l'idée générale, le rôle d'Etat) aux Expositions précédentes. Pourtant les

${ }^{1}$ CTACOB, Владимир Васильевич, Наши итоги на всемирной выставке, URL:

< http://az.lib.ru/s/stasow_w_w > [consulté le 5 avril 2014].

2 Наследие Александра Николаевича Бенуа, URL:< http://www.benua-memory.ru > [consulté le 5 avril 2014].

3 АВЕТИСЯН, Ашот Джеймсович, Традиции русского зодчества в архитектуре павильонов России на всемирных выставках іп Российская Академия архитектуры и строительных наук, Вопросы всеобщей истории архитектуры: Материалы международной научной конференции 3о-31 мая 2013, Москва, 2013.

4 MЕЗЕНИН, Николай А., Парад всемирных выставок, URL:

< http://nplit.ru/books/item/foo/soo/zooooo91/sto05.shtml > [consulté le 20 avril 2014].

5 ШПАКОВ, Валерий Н., История всемирных выставок, Москва, АСТ Зебра Е, 2008. 
publications portent plutôt le caractère idéologique que purement scientifique, descriptif qu'analytique, apologétique que critique. Selon eux, tous les pavillons présentaient bien l'identité de la Russie et des Russes. Parmi des plus significatives publications on peut noter Russie aux Expositions universelles: les meilleurs pavillons depuis 150 ans ${ }^{6}$ et Histoire de la participation de Russie aux EXPOS7. Un des derniers articles est Pavillons de la Russie des Expositions Universelles: la construction et l'architecture de l'universitaire Vladimir Frolov8.

L'auteur de l'article propose l'interprétation du sujet du point de vue de l'imagologue, la science interdisciplinaire, étudiant la perception des autres peuples, pays, cultures. L'auteur est le membre de l'association des chercheurs de l'histoire de la perception des cultures de l'Institut d'histoire russe de l'Académie des Sciences9. Dans le cadre imagologique les questions de l'identité, des stéréotypes nationaux, de l'image transmise et reçue occupent la première place dans l'histoire des pavillons russes des Expositions Universelles.

\section{La participation de la Russie aux Expositions Universelles}

L'Empire russe a rencontré de graves difficultés pour sa représentation aux expositions. Dès l'époque de Pierre Le Grand, son identité se construisait autour de l'idée de sa direction européenne du développement ce qui a emmené au milieu du $\mathrm{XIX}^{\mathrm{e}}$ siècle à une rupture immense entre sa culture populaire (traditionnelle) et sa culture officielle (occidentalisée). L'évolution des pavillons russes est en même temps l'histoire des recherches de sa propre identité exceptionnelle.

La Russie a pris part à toutes les expositions sauf celle de Paris de 1855 car elle se trouvait en état de guerre avec la France. Son activité d'exposition dépendait du lieu d'accueil, du financement et de l'état des relations internationales. Si au cours de la première exposition de 1851 la section russe ne faisait voir que quelques participants particuliers, à l'Exposition de 1900 à Paris («l'Exposition du siècle») la Russie disposait des plus grands espaces. Cette année-là, ayant envie de fêter son alliance avec la France, le gouvernement russe a investi des millions à l'exposition et à la construction des pavillons nationaux.

6 ЖИДКАЯ, Анна, Россия на всемирных выставках: лучшие павильоны за 150 лет, URL: < http://obzor.westsib.ru/article/368101 > [consulté le 25 juin 2014].

7 История участия России в ЭКСПО, URL:

< http://expo2010.formika.ru/about/history.php > [consulté le 25 juin 2014].

8 ФРОЛОВ, Владимир П., Павильоны России на Всемирных выставках: строительство и архитектура, in Вестник МГСУ, 6, 2013, Сc. 42-49.

9 URL: < http://rosmir.iriran.ru > [consulté le 25 juin 2014]. 
Le problème de l'étude des pavillons d'exposition est l'ignorance de leur statut d'une pièce exposée par de nombreux «rapports» officiels, «aperçus», «catalogues», décrivant le contenu des sections, mais non leur aspect extérieur. Les guides trouvaient important de faire les visiteurs s'orienter parmi des centaines d'objets exposés donc les pavillons n'ont pas reçu d'appréciations détaillées. Les commentaires des visiteurs restent les sources essentielles de l'étude des pavillons d'exposition. En général, ils appréciaient les pavillons du point de vue de l'incarnation de l'image de la Russie avec laquelle ils associaient le pays dans leur imagination.

Il faut dire que les organisateurs de la participation russe aux expositions étaient orientés vers les goûts et les attentes du public européen en lui proposant quasi fausse image de la Russie, image construite artificiellement.

De notre point de vue, c'est «l'Isba russe» de Vassiliï Gromov (1798-1869), commerçant du bois, qui a contribué d'une façon décisive à la création de la conception et du style des pavillons nationales. Pour la première fois elle a été présentée à l'exposition de 1867 à Paris. L'isba faite de rondins de bois et décorée de dentelles de bois était initialement un projet publicitaire de l'entreprise de Gromov, mais compte tenu du grand intérêt de la part du public elle est devenue un objet représentatif, déménageant d'une exposition à l'autre. L'isba est construite suivant l'esthétique du style russe ou «à la russe» qui a monopolisé depuis lors la décoration des pavillons russes aux Expositions Universelles.

Leurs auteurs représentaient un cercle relativement étroit de partisans des idées de A. M. Gornostaév (1808-1862), professeur d'architecture de l'Académie des Beaux-Arts dont la perception romantique de l'art du peuple s'est exprimée par l'intérêt passionnant envers l'art populaire. Après la mort du professeur ses disciples et collègues se sont réunis autour de la revue «Zodtchï̈» (architecte en russe) et de l'almanach «Motifs de l'architecture russe» où ils ont publié leur credo et leurs projets.

\section{Le style pseudo-russe en architecture: principes, particularités, évolution}

Ce courant architectural est assez difficile à classer et à évaluer. Les chercheurs le reportent à l'éclectisme (renaissance et combinaison des éléments architecturaux des temps passés) et à l'historisme (reproduction précise des modèles architecturaux du passé). Dans la littérature étrangère il est nommé le style russe ce qui est aussi général, vague et incorrect, car tout style ayant une originalité ou une singularité peut être 
appelé national, par exemple «baroque moscovite» de la fin du XVII siècle ou «moderne du nord» du début de XX siècle. Même si on se met d'accord d'appeler «style russe» le mouvement de l'art du XIX siècle apparu à la suite du nationalisme et de l'intérêt pour l'histoire de la Russie ancienne, il n'est pas homogène.

La mode architecturale de cette période, plus que tout autre genre de l'art, se déterminait par l'idéologie plus que par le goût artistique. Aux années 1830-1850e le «style russe» se développait dans le cadre de l'idéologie officielle de Nicolas I (autocratie + orthodoxie) sous le nom du style russe-byzantin (exemple - la cathédrale du Saint-Sauveur à Moscou). Aux années 1860-1870e, parallèlement au précédant, se formait le style pseudo-russe (folklorique) né de l'intérêt des intellectuels libéraux et révolutionnaires envers la culture populaire. Aux années 1880-1890e, dans l'atmosphère de l’idéologie politique réactionnaire des autorités il a été formé le style russe historique comme la copie exacte ou l'imitation des formes architecturales du XVII siècle (Musée historique sur la Place Rouge). Enfin au début du XX siècle révolutionnaire du point de vue politique et décadent du point de vue culturel - on a vu se former le style néo-russe («moderne du nord», art-déco) inspiré des églises anciennes du Nord Russe. L'époque du style pseudo-russe que nous étudions a été très courte en Russie mais elle a duré de manière artificielle grâce à l'accueil chaleureux aux Expositions Universelles.

D'autres noms de ce style sont plutôt journalistiques qu'académiques - «style de coq», «ropetovchina» (selon le nom du leader de ce courant - Ivan Ropete), «bérendéévchina» (au nom de Bérendeï, tsar légendaire de l'opéra de Rimski-Korsakov «Snégourotchka»). Tous ces noms ont une nuance négative à cause de la négligence de ses adeptes vis-a-vis des canons architecturaux.

Les principes constructifs et esthétiques de ce style pseudo-russe sont:

- le bois en tant que la matière première qui multiplie des moyens décoratifs et limite en même temps d'autres moyens architecturaux. A cause de la fragilité de ce matériel la Russie n'a conservé que quelques rares monuments de l'architecture de bois c'est pourquoi à la différence des autres pays-participants des Expositions Universelles les façades russes n’ont été ni copies ni imitations mais des créations originales des architectes contemporains;

- nombreux éléments non-architectutaux - détails et ornements des broderies (surtout celles des serviettes), des vêtements, des vaisselles, des articles ménagers;

- attention vers la culture populaire traditionnelle avant le règne de Pierre Le Grand et même avant du baptême de la Russie ce qui contredisait l'identité officielle de l'Empire russe en tant qu'un état européen et chrétien; 
- aspiration intentionnelle, ostentatoire, grotesque à l'originalité, l'abandon des canons académiques, des idées de l'harmonie et de l'équilibre ce qui engendrait des constructions asymétriques, avec de grands toits, balcons, tours disproportionnés;

- manque du sens de la mesure dans l'ornement et la colorisation des bâtiments;

- sacrifice des fonctions et de la commodité intérieure au profit de la beauté extérieure des édifices, par exemple, un puits entièrement orné de bois sculpté, un cheminée en bois, l'escalier à l'étage inexistant. Il n'est pas étonnant que la plupart des projets ne soient jamais réalisés.

Toutes ces particularités du style pseudo-russe répondaient bien aux attentes des visiteurs des Expositions Universelles. Ils admiraient des murs, sont bâtis des troncs entiers et sans clous, de cadres, de chambranles et de corniches sculptés, de l'ornement païen des fleurs, des oiseaux et des animaux, du décor multiforme et multicolore, de bulbes et de tentes de toits. Tous les excès et les erreurs de style ont été attribués à l'identité nationale des Russes - des demi-Asiatiques semi-sauvages. Les visiteurs étrangers croyaient sincèrement que les bâtiments présentés à leur attention sont les copies et les imitations justes des oeuvres de la vieille Russie.

En plus, le succès aux expositions a contribué à la propagande et au développement de ce style en Russie. Ayant commencé à construire de grands pavillons dans les capitales européennes les membres du groupe ont passé des projets de puits, de bains et de rotondes à ceux de grandes constructions (supermarchés, écoles, théâtres). D’autre part, en analysant l'expérience des Expositions Universelles, les organisateurs des expositions en Russie se sont mis à leur commander des pavillons pour les expositions et les foires régionales.

\section{Les pavillons russes en tant que l'expression de l'identité nationale aux yeux des visiteurs étrangers}

Observons l'histoire des pavillons russes aux Expositions Universelles du point de vue de l'image du pays présentée aux visiteurs et de sa perception par les étrangers.

A l'Exposition de 1851 à Londres les exposants russes, sans le soutien de l'Etat, ont décoré leur coin dans le Palais de Cristal de manière peu expressive, dans le style classique. La soie et les fourrures, les bijoux, les vases et les meubles de la malachite 
donnaient aux visiteurs l'impression de l'exotisme oriental qui fortement contrastait avec «les souvenirs du siècle de Pierre le Grand», selon l'un des eux.10

En effet, la prédominance des articles de luxe dans l'exposition principale pendant des années 1850-1890 est devenue le trait caractéristique de la participation russe ce que contredisait l'idée du concours mondial aux domaines industriel et technique et liait la Russie avec des pays pre-industriels. À l'avis des observateurs russes, cela non seulement déshonorait le pays du point de vue du progrès de la civilisation, mais aussi créait l'image fausse de la Russie chez les étrangers. «Regardez que se passe aux Expositions Universelles dans la section de notre patrie. - S’indignait un des eux. Chacun peut imaginer que chez nous il n'y a rien et personne, sauf des comtes, et des princes, et des millionnaires. Tout cela, doit être, du monde magique, où les gens se nourrissent des frits oiseaux de paradis, et s'habillent des plumes de ces oiseaux. Et c'est notre vraie Russie, dont nous connaissons et voyons? ${ }^{11}$.

A l'exposition de 1862 à Londres l'Empire russe s'est présenté officiellement. Sous Alexandre II, le réformateur libéral, la Russie a voulu manifester tous ses succès du développement capitaliste et son attachement à l'esthétique européenne. Son pavillon au Palais de l'industrie avait le même style classique, les colonnes corinthiennes strictes ornaient son entrée. Le correspondant d'un journal de Moscou soulignait comme la dignité de l'exposition russe ce que tous les objets «ont le sceau de la civilisation européenne supérieure et du goût artistique raffiné ce que promet beaucoup pour le futur de l'industrie russe» ${ }^{12}$. Pourtant les visiteurs a semblé ne pas remarquer la section russe et l'ont trouvé ennuyeuse. Parmi les pièces exposées se trouvaient des modèles de l'Eglise orthodoxe et de l'isba en bois vernie. Inattendu l'isba a attiré l'attention du public et de la presse.

${ }^{10}$ Российская империя в зеркале Всемирных универсальных выставок. URL: < http://sergeytsvetkov.livejournal.com/134837.html > [consulté le 5 avril 2014].

${ }^{11}$ CTACOB, Владимир Васильевич, Наши итоги на всемирной выставке, URL:

< http://az.lib.ru/s/stasow_w_w/text_1878_nashi_itogo_na_vsemirnoy_vystavke.shtml > [consulté le 5 avril 2014].

${ }_{12}$ «Всемирная выставка в Лондоне», in История выставочно-ярмарочной деятельности, 1862, URL: < http://istoriya-vystavok.narod.ru/index/o-5 > [consulté le 5 avril 2014]. 
Fig. 1. Le petit pavillon au style de l'isba russe de l'Expo-1862'13.

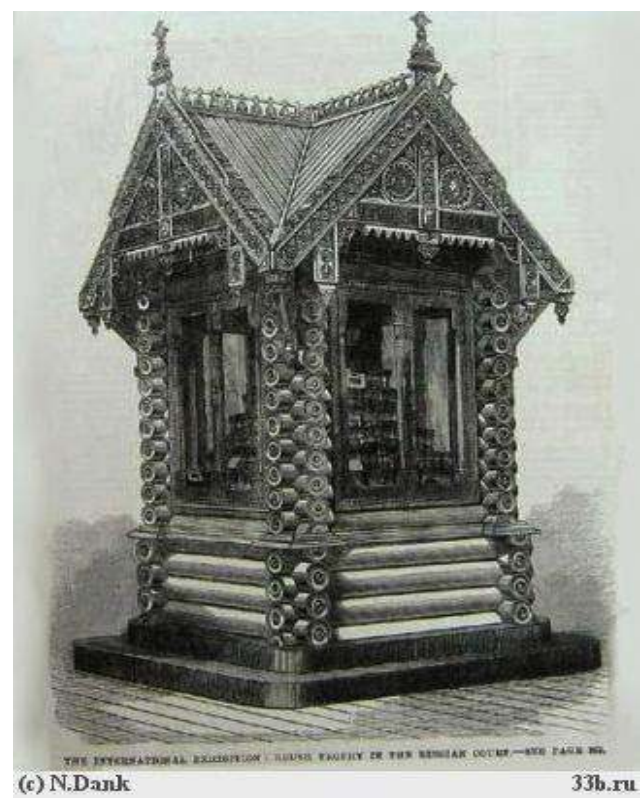

En réponse à l'intérêt du public, à l'Exposition de 1867 à Paris la façade de la section russe au Palais Omnibus a été décorée dans le "style paysan», même si c'était encore trop conventionnel et trop modeste (sous la direction de l'architecte parisien Bénard): une visière au-dessus de l'entrée et quelques colonnes en bois. Derrière la façade paysanne on exposait toutes les mêmes fourrures, bijoux et articles de luxe. C'est donnait l'étrange discordance de sens et de style entre l'extérieur et l'intérieur de la section russe. Hippolyte Gautier, le guide professionnel, réfléchissait dans la section russe: «De toutes les expositions étrangères qui ont trouvé place au Champ de Mars, celle de la Russie est peut-être celle qui a le plus de saveur, qui attire le plus par je ne sais quoi d'étrange et de mystérieux. Le Russe est notre voisin, il vit chez nous, de notre vie, il est aussi Parisien qu'homme du monde: de la notre étonnement quand nous le voyons chez lui, quand ses arts, ses productions nous montrent ce singulier mélange de l'Orient et de l'Occident, de la civilisation et de la barbarie, des robes de soie et des peaux qui servent de vêtement à l'habitant du Caucase; jusque dans les salles qui renferment l'exposition russe, toutes en bois sculpté, de dessin le plus capricieux, des couleurs les plus fantastiques, il y a quelque chose de singulier: on sent qu'on est plus près de Byzance que de la Sibérie» ${ }^{14}$.

${ }_{13}$ URL: < http://weblog.33bru.com/blog.kommentar.24024063.html > [consulté le 5 avril 2014].

14 GAUTIER, Hippolyte, Les curiosités de l'exposition universelle de 1867, Paris, Ch. Delagrave, 1867. p. 115 . 
Fig. 2. La façade de la section russe de l'Expo-186 $7^{15}$.

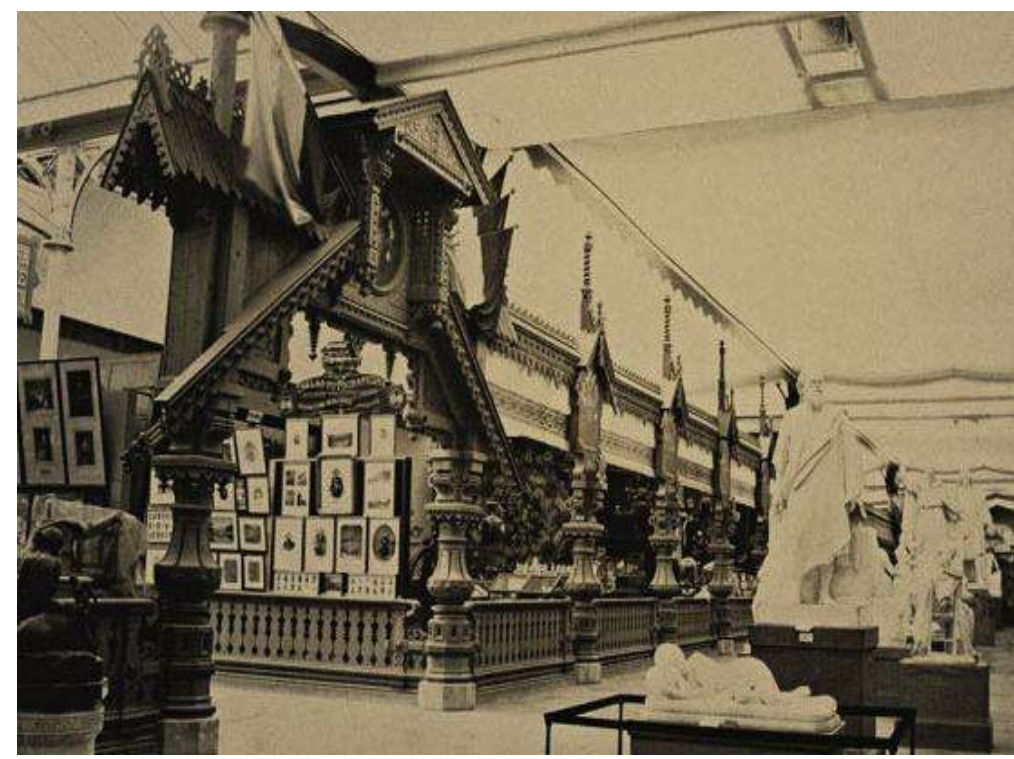

L'Isba de Gromov a été exposée à l'extérieur du Palais. Elle a été bâtie par son architecte personnel G.I.Vintergalter, l'ami et l'adhérent du professeur Gornostaév. A Pétersbourg cette oeuvre a reçu la recommandation de l'Académie des Beaux-Arts. L’isba a été démontée et est arrivée à l'Exposition dans 2500 boîtes accompagnée de moujiks barbus.

Document 3. Le village russe de l'Expo-186716.

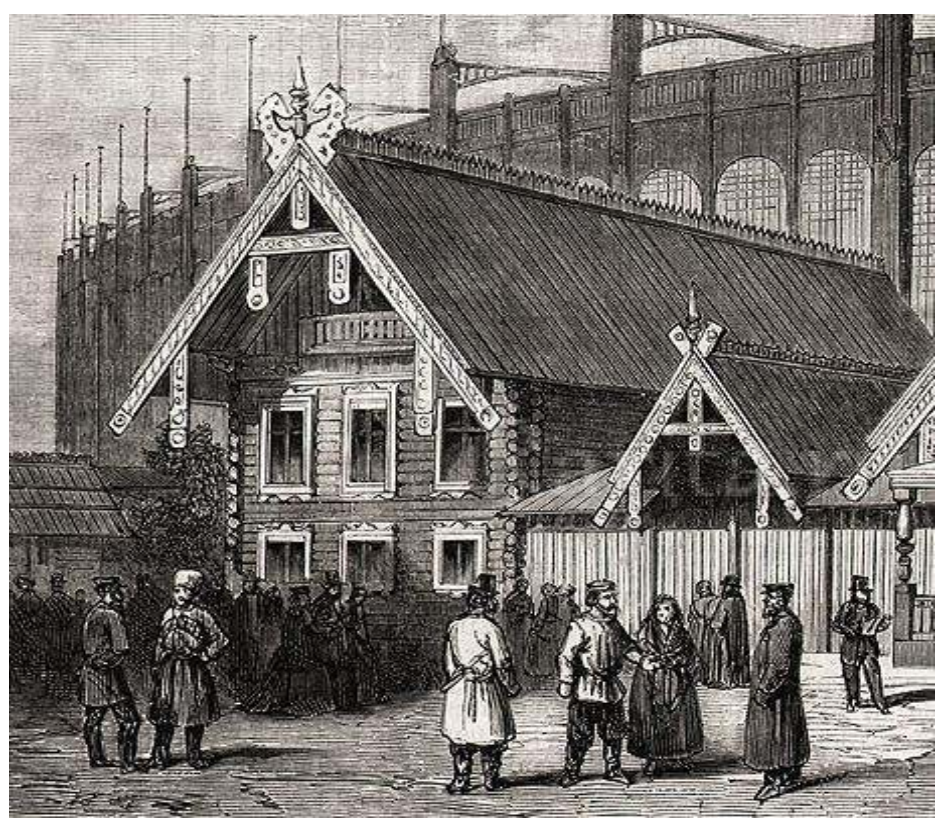

Elle a reçu la médaille d'argent du jury comme l'oeuvre d'art. Les visiteurs étrangers ne savaient pas comment classifier ce bâtiment - la cabane de paysans ou l'élégant

15 URL: < http://obzor.westsib.ru/article/368101 > [consulté le 5 avril 2014].

${ }^{16} \mathrm{URL}:$

<http://rarita.ru/catalog/gravyury/russkie_pavilony_na_vsemirnoi_vystavke_v_parije_v_186 7_g > [consulté le 5 juin 2014]. 
chalet. Selon un des eux, «si les paysans russes en ont de semblables il faut croire qu'ils ne sont pas trop a plaindre»17. Les guides ont consacré des chapitres entiers à l'isba et à ses intérieurs. Parfois leurs illustrations étaient le fruit des idées stéréotypées des peintres français sur les «moscovites». Ainsi dans l'Album de l'Exposition illustrée sur la gravure «Isba» nous voyons un enfant aux cheveux frisés jouant sur la peau d'un ours, un luth (!) sur le plancher aux pieds d'un moujik faisant prière avec une hache (!) à sa ceinture. Pourtant la revue soulignait sa compétence: «Nous nous faisons généralement une très fausse idée du caractère de certains peuples, entre autres du Russie, qu'il est convenu de considérer comme un barbare. Le Russe est gai et joyeux, sujet à l'enthousiasme, crédule jusqu'à la naïveté, bavard, fou de chants et de danses, superstitieux, brave à la guerre, et impassible devant la mort» ${ }^{18}$. Telles conclusions sur l'identité nationale du peuple russe étaient faites par les observateurs de "l'Isba" de Gromov. Mais même les étrangers ont deviné que cette maison est trop luxueuse pour les simples gens: «Elle nous offrait plutôt une reproduction élégante des habitations de paysans russes, que leur type réel et tel qu'il se rencontre le plus souvent dans cet empire», - lisons dans un ouvrage de référence. ${ }^{19}$

L'exposition de 1873 à Vienne est devenue la vraie triomphe de Russie sur le fond de l'intérêt décroissant des Anglais et de la participation réduite de la France après sa guerre avec Bismarck. L'architecte de la section russe était Ippolito Monighetti (18191878), un des rédacteurs de la revue «Zodtchiï», du foyer de pseudo-russe style. Il a exposé trois objets du même type: la façade de la section russe à l'intérieur du Palais de l'industrie entre les sections de l'Egypte et de la Turquie, l'isba de Gromov et le pavillon de l'Empereur sous la forme d'une partie du maison de boyard - dans le parc avec les mêmes voisins. Il est possible que ce voisinage aussi bien que la passion de Monighetti pour l'architecture de l'Asie renforçaient la perception de l'exposition russe par les visiteurs comme de l'exotisme oriental.

17 GAUTIER, Hippolyte, op.cit. p. 46.

18 RICHARD, Gabriel, L'Album de l'Exposition illustrée. Histoire pittoresque de l'Exposition universelle de 1867, Paris. C. Schiller, 1867. p. 291.

19 NORMAND, Alfred, L'architecture des nations étrangères : étude sur les principales constructions du parc à l'Exposition universelle de Paris, Paris, A. Morel, 1870. p. 15. 
Fig. 4. Maison de paysan russe et le pavillon de l'empereur de l'Expo-187320.

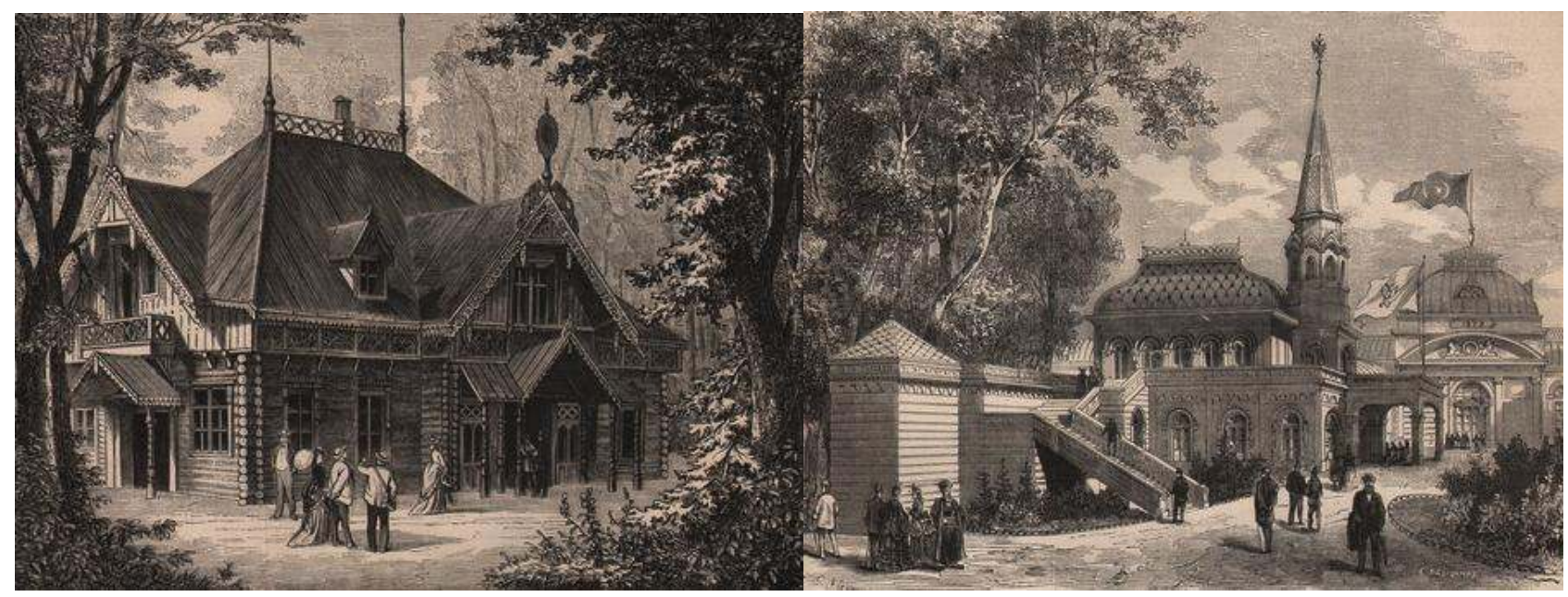

Selon un guide, «l'aspect du pavillon russe est tout à fait grandiose» ${ }^{21}$. Parmi tous les bâtiments russes l'attention du public était attirée vers l'isba: «Rien de coquet et d'élégant comme l'Isba russe, qui donnera aux visiteurs de l'Exposition une idée de l'architecture et de la construction employées par le paysan pour se maison» ${ }^{22}$.

Les goûts du public américain étaient complètement inconnus aux participants russes. Ils sont arrivés en retard à l'Exposition de 1876 à Philadelphie, les organisateurs leur ont donné des meubles ordinaires. C'est pourquoi la section russe en Amérique était plus pauvre des impressions identitaires par rapport aux autres participants.

Pourtant même ses quelques vitrines de style russe ont suffi pour attirer l'attention des visiteurs. Louis Simonin, l’ingénieur français, a écrit pour «Revue des Deux Mondes»: «La Russie se fait également remarquer dans les bâtiments du parc de Fairmount; sa double physionomie de nation à la fois européenne et asiatique y apparaît nettement» ${ }^{23}$. Un chroniqueur américain a critiqué «la manie», cette passion pour le folklore au sein de l'exposition industrielle:

20 URL: < http://www.worldfairs.info/expopavillondetails.php?expo_id=4\&pavillon_id=1604 > [consulté le 5 avril 2014] et URL:

< http://www.worldfairs.info/expopavillondetails.php?expo_id=4\&pavillon_id=1609 > [consulté le 5 avril 2014].

${ }^{21}$ L'exposition universelle de Vienne illustrée, 17, 1873, pp. 260-261,

URL: < http://babel.hathitrust.org/cgi/pt?id=umn.31951do25531617;view=1up;seq=206 > [consulté le 5 avril 2014].

${ }^{22}$ Maison de Paysan Russe, URL:

< http://www.worldfairs.info/expopavillondetails.php?expo_id=4\&pavillon_id=1604 > [consulté le 5 avril 2014].

${ }^{23}$ SIMONIN, Louis, Le centenaire américain et l'exposition de Philadelphie. // Revue des deux mondes, T. 17 1876, URL:

< http://fr.wikisource.org/wiki/Le_Centenaire_americain_et_l'exposition_de_Philadelhie > [consulté le 5 avril 2014]. 
The Russian section was a fruitful field, presenting in a striking manner the results of the revived taste for national forms and ornamentation as expressed in early Muscovite art and architecture that now prevails to a very marked extent among all classes of Russian society. This taste is a recent growth; but stimulated by an intense patriotism, and by the most exalted ideas of the future of the Slavic race, it is almost a mania, and bids fair to greatly modify if not entirely replace the taste for classic, renaissance, and modern European art 24 .

L’Espagne, notre voisine du pavillon, a apporté à Philadelphie une grandiose façade en bois. A l'Exposition américaine suivante ayant lieu en 1893 à Chicago les Russes ont amené une construction analogue, «l'entrée-vestibule» faite par Ivan Ropete (18451908). Elle a été composée de bois sculptés et vernis avec des tourelles carrées sur les coins. Les toits des tourelles étaient couronnés des aigles bicéphales. Toute la façade était colorée et recouverte de dentelles en bois. Les visiteurs et les organisateurs ont bien aimé cet objet magique. Après la fermeture de l'exposition elle a été offert à la mission orthodoxe russe aux Etats-Unis, ainsi «l'entrée-vestibule» s'est transformée en façade de l'église à Illinois, continuant d'affirmer l'identité nationale au-delà de l'océan.

24 INGRAM, Joseph S., The Centennial Exposition described and illustrated: being a concise and graphic description of this grand enterprise commemorative of the first centenary of American independence, Philadelphia- Toronto, Hubbard-A.H. Hovey, 1876. URL:

< https://archive.org/stream/centennialexposition\#page/470/mode/2up > [consulté le 5 avril 2014]. 
Fig. 5. La façade de la section russe à l'Expo-1893²5.

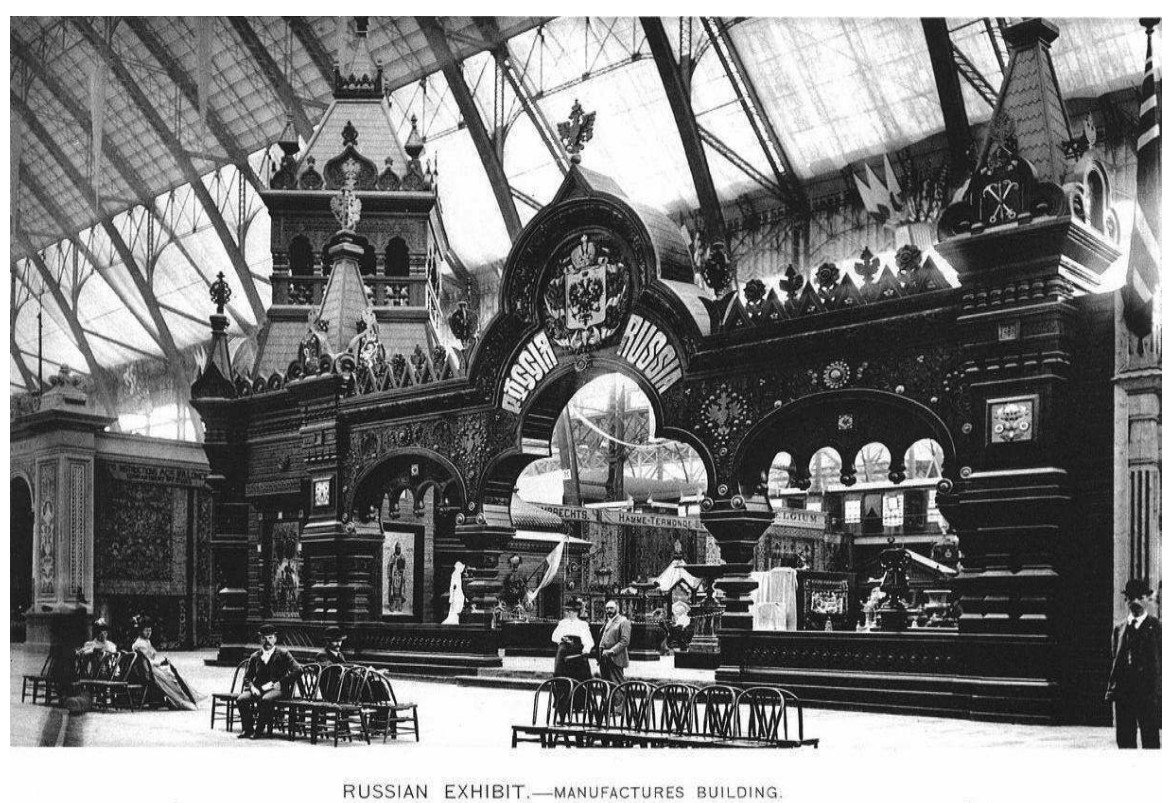

L'apogée du style pseudo-russe des pavillons nationaux est l'Exposition de 1878 à Paris et le déclin - l'Exposition de 1900 à Paris aussi.

Pour la «Rue des Nations» de l'Expo-1878 Ivan Ropete (anagramme de Petrov) a créé une façade asymétrique inspiré du palais de Kolomenskoé près de Moscou où Pierre Le Grand était né. Le bâtiment représentait une maison à rondins de bois colorés avec un haut toit surmonté d'un «kniazek» et d'un «kokochnik» (éléments décoratifs). De deux côtés du bâtiment central s'étendait la multitude des détails diverses: tours, passerelles, escaliers, toute sorte de fenêtres. L'ensemble était plein d'élégance, de l'impression de fête, de beauté exquise et de raffinement. Il a bénéficié d'un énorme succès. Puisque aux expositions précédentes la Russie faisait la propagande de l'identité traditionnelle par les façades sculptées de ses sections, dans une nouvelle construction de bois de Expo-1878 les spectateurs cherchaient des connus signes de l'isba, même si elle est immense. Ils ont décidé que le pavillon de Ropete est une habitation aristocratique, le mélange de l'isba de moujik et du palais de tsar: «C'est, en un mot, le type de la maison du boyard, appelée téréma» ${ }^{26}$.

25 URL:<http://humus.livejournal.com/2617017.html > [consulté le 5 avril 2014].

${ }^{26}$ Rue des nations. Pavillon russe, URL:

$<$ http://www.worldfairs.info/expopavillondetails.php?expo_id=25\&pavillon_id=2627 > [consulté le 5 avril 2014]. 
Fig. 6. Ropete, Ivan, Le projet du pavillon russe de l'Expo-187827.

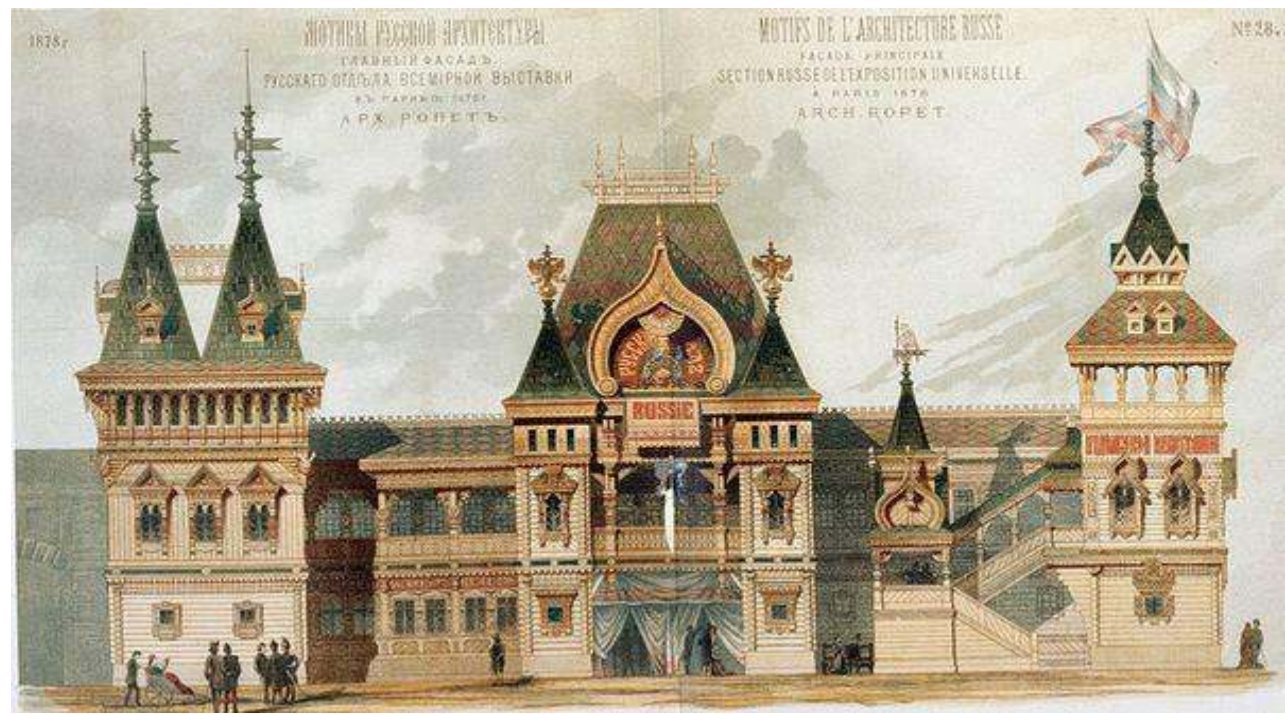

Selon l'opinion générale des visiteurs de l'Exposition, le pavillon national était original, talentueux, faisait l'honneur de la Russie et ornait la manifestation. H. Gautier et A. Desprez ont écrit dans le guide Les curiosité de l'exposition : «La façade russe peut se reconnaître de loin à sa masse imposante de bois étagé, découpé, festonné en tous sens, à ses hautes toitures aiguës, à ses encadrements bizarres de fenêtres, à la forme quasi-turque du tympan central. La couleur dont on a revêtu les ornements de bois, les découpures, les toits, a plutôt gâté qu'embelli ce monument; mais l'on a bien fait de l'en revêtir, si elle est historique. C'est dans tous les cas la plus sérieuse, la plus intéressante (façade) à étudier» ${ }^{28}$.

L'exposition parisienne de 1889 était consacrée au centenaire de la chute des Bourbons c'est pourquoi l'empire des Romanovs a renoncé à la participation officielle. Faute de ressources, la section russe est revenue sous le toit du Palais de l'industrie, elle était petite et modeste présentant les mêmes curiosités touristiques - samovars, icônes, caviar... et $8 \mathrm{~m}^{3}$ de terre.

Le rôle inattendu et substantiel dans la propagande du style russe en 1889 appartenait à l'exposition «Histoire de l'Habitation humaine» de Charles Garnier. Elle est devenue le résultat de ses recherches scientifiques. En qualité de l'habitation russe Garnier a choisi la maison de l'aristocrate médiéval (le palais de bois des boyards, en fait le même palais Kolomenskoé). L’auteur l'a rangé dans la partie «la civilisation Byzantine et son influence dans l'Europe», ainsi en soulignant les motifs orientaux du bâtiment. À son

${ }_{27}$ URL: < http://wood.totalarch.com/node/98 > [consulté le 5 avril 2014].

28 GAUTIER, Hippolyte, DESPREZ, Adrien, Les curiosités de l'exposition de 1878 : guide du visiteu, Paris, Ch. Delagrave, 1878, p. 37. 
avis, le style russe dans l'architecture est un mélange du style byzantin - mais le plus luxueux et aristocratique, et du slave style traditionnel ${ }^{29}$.

Fig. 7. Maison russe au XV siècle (entre les bâtiments byzantin et arabe) / Histoire de l'habitation humaine à l'Expo-188930.
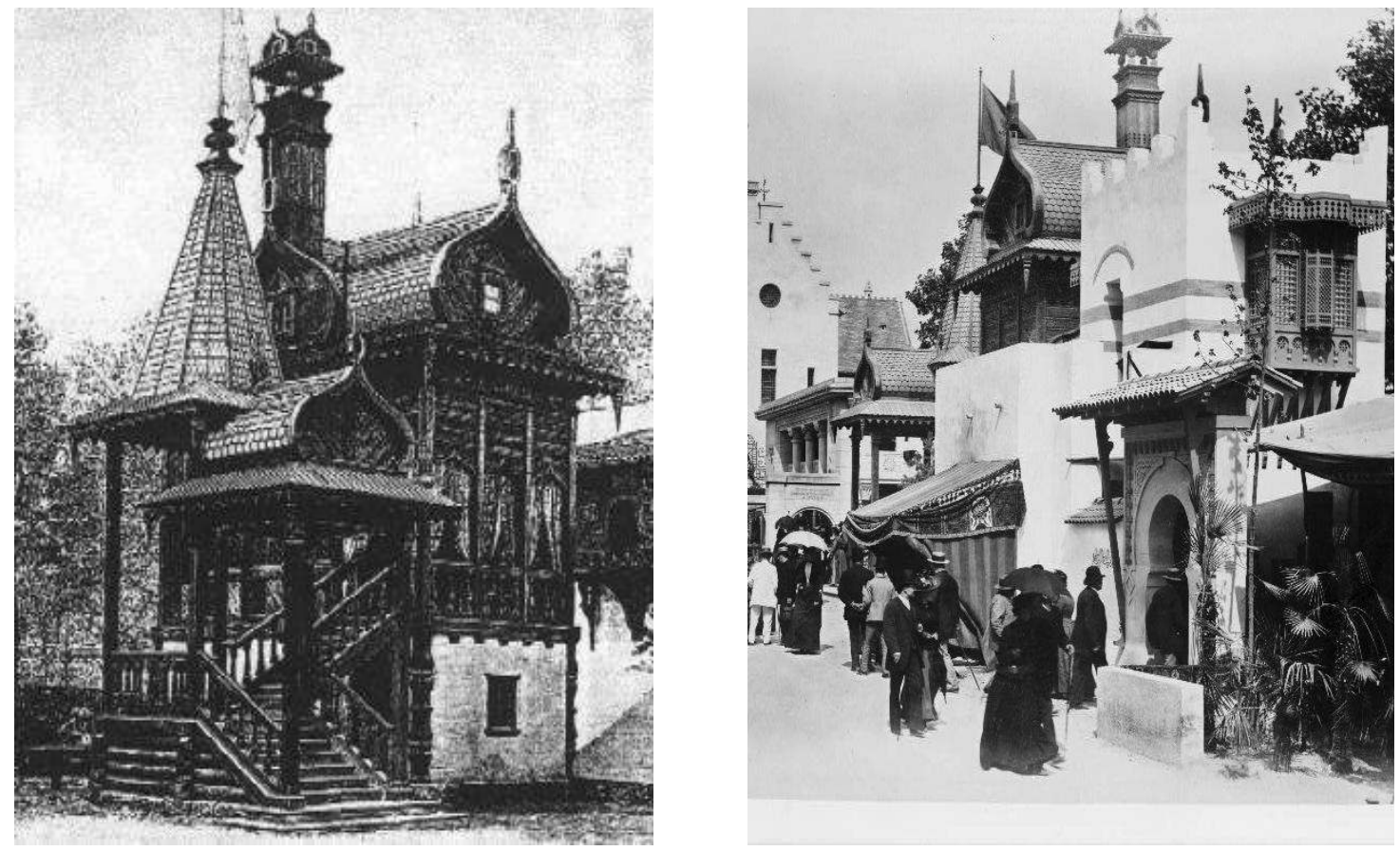

Pour l'Exposition-1900 à Paris, à l'époque de «l'Entente cordiale» entre deux pays, la Russie se préparait longtemps et sérieusement. Un projet du pavillon en style pseudo-russe, déjà familier aux visiteurs européens, a été proposé par Victor Vasnetsov (1848-1926), célèbre peintre et illustrateur des contes slaves. Pourtant ce projet a été rejeté parce qu'il n'incarnait pas l'idée de l'Empire.

Finalement c'est le Pavillon des Confins Russes rempli de tapis de Boukhara, de défenses de mammouth, de chèvres de montagne et d'ours polaires est devenu le bâtiment principal. Il a été fait en pierre ce qui détruisait l'esthétique du style pseudorusse destiné aux constructions en bois. L'auteur de ce projet est Robert-Fridrich Meltser (1860-1943), architecte de la cour impérial et partisan des idées du professeur Gornostaév. Meltser était connu pour sa capacité de stylisation et de mélange des styles le plus souvent orientaux (chinois, indien, mauresque). Dans les silhouettes du pavillon les visiteurs étrangers voyaient le Kremlin de Moscou. «Cette évocation du Kremlin

29 GARNIER, Charles, AMMANN, Auguste, L'Habitation humaine, Paris, Hachette, 1892, p. 702.

30 URL: <_http://www.uefexpo.ru/?id=44965 > [consulté le 5 avril 2014] et URL:

$<$ http://humus.livejournal.com/1369937.html > [consulté le 5 avril 2014]. 
rappelle l'architecture de ce palais intimement lie à l'histoire de la grandeur croissante de la sainte Russie et rappelle les gloires des tzars, pères de ce peuple immense qui leur doit sa prospérité actuelle précédant un avenir plein de superbes promesses» ${ }^{31}$.

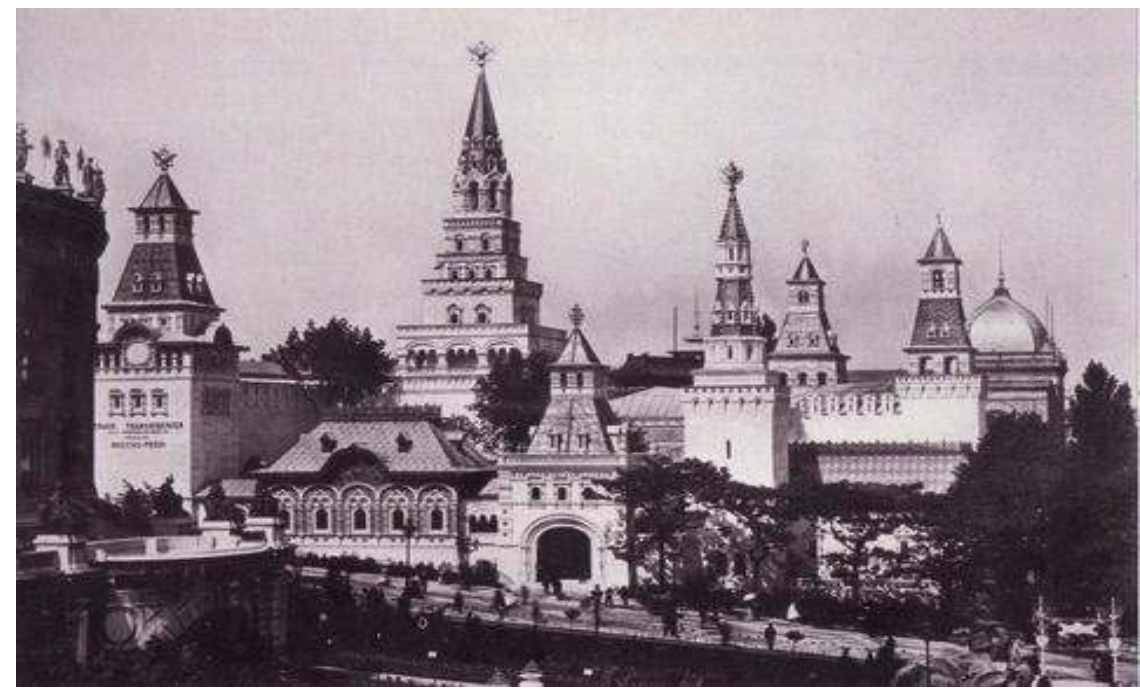

Fig. 8. Le pavillon des Confins Russes à l'Expo-190032.

L'objectif de la présentation de l'identité officielle au moyen du pavillon d'Exposition a été réalisé tout à fait. Les spectateurs étrangers le classifiaient sans hésitations comme le palais. Même les critiques-compatriotes du style pseudo-russe ont reconnu que le pavillon avait l'aspect «princier»33. Ayant la vue du Kremlin, il a été consacré aux confins asiatiques de l'empire - le Caucase, l'Asie centrale, la Sibérie - ce qui a permis aux visiteurs de le surnommer le palais sibérien. Grâce à ce phénomène la conscience européenne a fini par unir le style russe des pavillons d'exposition à l'exotisme oriental; l'identité russe était perçue comme asiatique ou, au moins, comme la civilisation mixte.

Le fossoyeur du «style russe» s'est caché dans une ruelle sombre entre le Palais sibérien et le Palais Trocadéro.

${ }^{31}$ Exposition universelle de 19oo: les plaisirs et les curiosités de l'exposition. Paris, Chaix, 1900, p. 272.

${ }^{2}$ URL: < http://www.worldfairs.info/expopavillondetails.php?expo_id=8\&pavillon_id=57 > [consulté le 5 avril 2014].

33 БЕНУА, Александр, Воспоминания, URL:< http://www.benua.su/book_4_g38_1/ > [consulté le 5 avril 2014]. 
Fig. 9. Le village russe à l'Expo-190034.

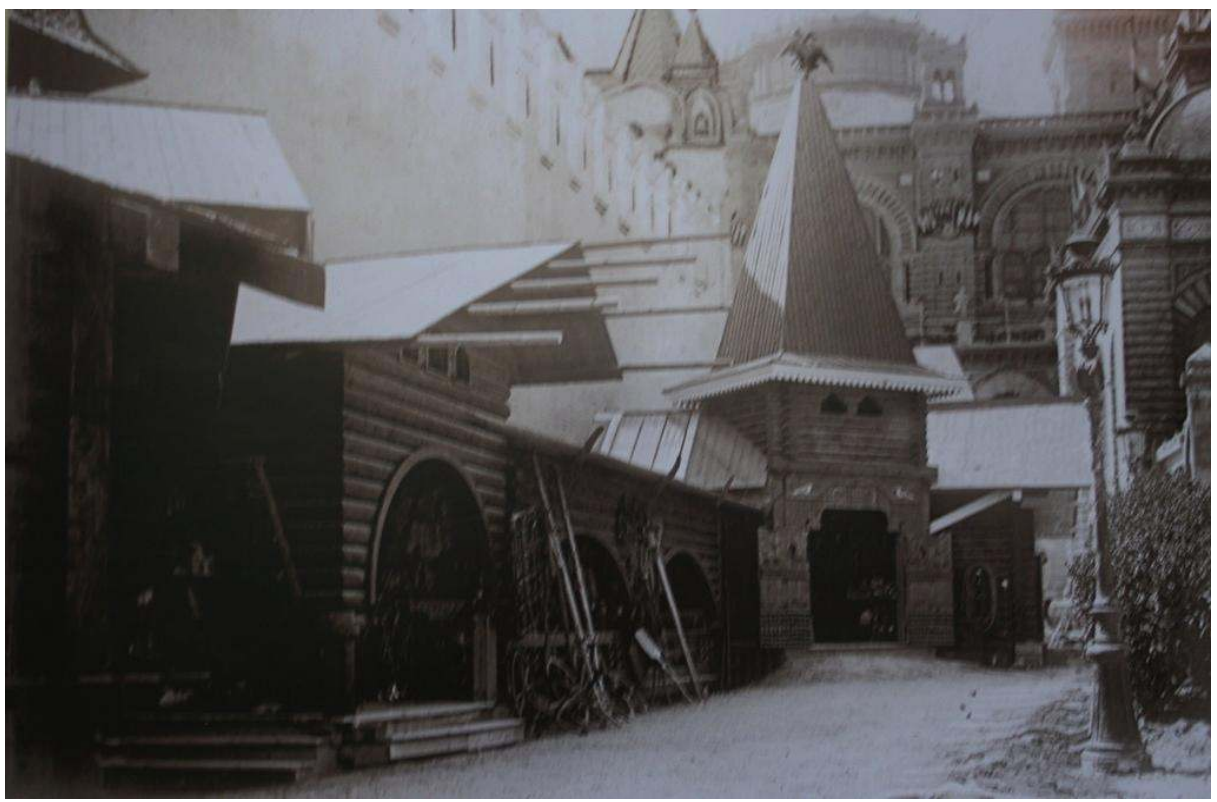

C'était la section artisanale (le village russe). Ce petit pavillon ne représentait pas une stylisation, une imitation et un emprunt de la culture de Russie ancienne, c'était sa révision originale à l'aide des moyens du nouveau courant d'architecture - le moderne. Les organisateurs de cette section, le peintre K. Korovine et l'architecte I.Bondarenko, ont interprété autrement le thème de l’isba russe, auquel le public s'était déjà accoutumé. Mais leur version de l'image de Russie était encore une variante de l'identité artificielle, fictive dont l'exemple le plus édifiant est la matriochka exposée pour la première fois (créée la veille à partir d'un jouet japonais) et devenue depuis lors le symbole principal de la culture russe dans l'imagination des étrangers.

\section{Conclusion}

La présentation des pavillons nationaux aux Expositions Universelles était la démonstration de l'originalité des pays-participants, le défi aux idées occidentales du progrès commun et du monde homogène.

Le design des pavillons russes des Expositions Universelles était le phénomène unique de l'architecture nationale et mondiale. On ne peut pas de classer son style en cadre des courants de l'architecture contemporaine, puisque il n'était pas architectural

34 URL: <http://cultobzor.ru/2014/o4/kollektsiya-morozova-eksponat-1/17-247> [consulté le 5 juin 2014]. 
ni par sa destination, ni par ses éléments. A la différence de l'éclectique européen, les architectes de «pseudo-russe» n'avaient pas des anciens modèles pour leurs combiner. La source de l'inspiration pour les auteurs des pavillons nationaux est devenu l'art décoratif et artisanal (les vêtements, les meubles, la vaisselle, la broderie). La compréhension de la fragilité et du caractère provisoire des bâtiments exposés, leur caractère représentatif dans le format des expositions universelles donnaient une grande liberté aux architectes pour la création des façades et la décoration des intérieurs des pavillons, c'est pourquoi les questions de l'harmonie, du goût, du style ont cédé aux critères de la vivacité, de l'attraction, de l'altérité - au «style de coq».

Pendant quelques décennies le cliché des pavillons des Expositions se formait par les efforts des architectes du cercle du professeur de l'architecture à l'Académie des beaux-arts A.M. Gornostaév.

L'extérieur des pavillons est devenu pour le public étranger l'expression concentrée de la russe caractère et culture, de l'identité nationale de la Russie. Notre recherche a montré que comme le style de l'architecture «à la russe» était le fruit de la fantaisie de ses auteurs, l'identité nationale affirmée par lui était fausse, artificielle, ne correspondait pas ni l'idéologie officielle de l'Empire, ni la mentalité de l'élite culturelle - profondément européanisée, ni le mode de vie - chrétien et modeste - du peuple russe.

$\mathrm{Au}$ début du XX siècle les images de l'isba traditionnelle et du bâtiment officiel remplaceront l'un l'autre comme les symboles de la compétition des deux identités russes (populaire et étatique), mais ils se réaliseront par les moyens des styles purement européens: de l'art-déco (Glasgow, 1901), du classicisme (Turin, 1911), du constructivisme (Paris, 1925).

Quelle image produisait la Russie par ses expositions? A notre avis, assez contradictoire.

Premièrement, il faut constater le contraste entre l'extérieur folklorique les pavillons russes et leurs intérieurs, plein des produits industriels et des innovations modernes. Par exemple, dans l'isba de Gromov (1867) s'installait l'écurie bien équipée, dans très joli pavillon d'Ivan Ropete (1878) se trouvait la direction de la section russe et son service postal, dans le palais des Confins russes, imitant l'architecture du XVII siècle (1900), s'installaient l'orchestre, jouant des marches et des polkas, et deux expositions du Transsibèrien avec le train de plaisir.

Deuxièmement, l'extérieur des pavillons russes du XIX siècle était le résultat sommaire des influences et des goûts des organisateurs de la participation du pays aux Expositions Universelles, qui se trouvaient sous l'influence (pour ne pas dire sous la 
pression) de la réaction des visiteurs étrangers. L'Europe, à la recherche de sa propre identité, ne voyait pas (consciemment ou inconsciemment) la Russie comme le pays moderne et occidental, le public attendait voir l'exotisme bizarre des coins perdus primitif, sauvage, asiatique. Le succès chez visiteurs et le bruit dans la presse fixait la conception des pavillons russes, la succession des façades des pavillons de l'Exposition à l'Exposition donnait au public l'impression d'observation de la véritable identité russe.

Troisièmement, la Russie tsariste de cette époque n'avait pas de grave crise identitaire. Elle se considérait comme le pays occidental, comme l'empire chrétien. Le nationalisme dans l'idéologie et dans la culture russe avait le même caractère qu'en Europe. Le style pseudo-russe était un des courants de ce mouvement, en plus il n'était pas trop populaire. Notamment le succès des pavillons des Expositions Universelles a donné le certain prestige et la popularité à ce style de coq. Pourtant le sens identitaire a été donné aux pavillons russes par nos contemporains, dont la conscience historique construit le mythe de la Russie ancienne - avant l'URSS -, pastorale, patriarcale, idyllique. L'image douce des façades d'expositions, surtout d'Ivan Ropete et de l'Expo1900 illustre ce mythe sur des centaines des pages des périodiques et des sites de l'Internet. 


\section{* L'auteur}

Olga Kazakova est Docteur en Histoire. Sa thèse Les Etats-Unis devant l'opinion publique russe des années 1850-1860: des socio-culturels aspects de la perception a été soutenue au mois de décembre 2000 à l'Université d'Etat d'Orel. Son intérêt principal est l'imagologie des relations internationales. Surtout spécialiste du XIX siècle elle étudie les différentes institutions et formes de la présentation et de la perception de l'image de l'Autre. Actuellement elle s'occupe de l'image de la Russie à travers des Expositions Universelles.

URL: < http://www.studistorici.com/progett/autori/\#Kazakova >

\section{Per citare questo articolo:}

KAZAKOVA, Olga, «Les pavillons russes aux Expositions Universelles du XIXe siècle: expression de l'identité qui n'a jamais existé», Diacronie. Studi di Storia Contemporanea : Le esposizioni: propaganda e costruzione identitaria, 29/6/2014,

URL: < http://www.studistorici.com/2014/6/29/kazakova_numero_18/ >

Diacronie Studi di Storia Contemporanea $\}$ www.diacronie.it

Risorsa digitale indipendente a carattere storiografico. Uscita trimestrale.

redazione.diacronie@hotmail.it

Comitato di redazione: Jacopo Bassi - Luca Bufarale - Elisa Grandi - Deborah Paci - Fausto Pietrancosta - Matteo Tomasoni - Luca Zuccolo

Diritti: gli articoli di Diacronie. Studi di Storia Contemporanea sono pubblicati sotto licenza Creative Commons 2.5 .

Possono essere riprodotti a patto di non modificarne i contenuti e di non usarli per fini commerciali. La citazione di

estratti è comunque sempre autorizzata, nei limiti previsti dalla legge. 